2014-02

\title{
Attention, predictive learning, and the inverse base-rate effect: Evidence from event-related potentials
}

\author{
Wills, Andy
}

http://hdl.handle.net/10026.1/2981

10.1016/j.neuroimage.2013.10.060

Neurolmage

Elsevier BV

All content in PEARL is protected by copyright law. Author manuscripts are made available in accordance with publisher policies. Please cite only the published version using the details provided on the item record or document. In the absence of an open licence (e.g. Creative Commons), permissions for further reuse of content should be sought from the publisher or author. 
Running title: ATTENTION AND PREDICTIVE LEARNING

Attention, predictive learning, and the inverse base-rate effect: Evidence from eventrelated potentials

Andy J. Wills ${ }^{\mathrm{a}}$, Aureliu Lavric ${ }^{\mathrm{b}}$, Yvonne Hemmings ${ }^{\mathrm{b}}$, Ed Surrey ${ }^{\mathrm{b}}$

a. School of Psychology, Plymouth University, Drake Circus, Plymouth. PL4 8AA. United Kingdom.

b. School of Psychology, Exeter University, Exeter. EX4 4QG. United Kingdom.

To appear in NeuroImage 


\section{Abstract}

We report the first electrophysiological investigation of the inverse base-rate effect (IBRE), a robust non-rational bias in predictive learning. In the IBRE, participants learn that one pair of symptoms $(\mathrm{AB})$ predicts a frequently occurring disease, whilst an overlapping pair of symptoms (AC) predicts a rarely occurring disease. Participants subsequently infer that $\mathrm{BC}$ predicts the rare disease, a non-rational decision made in opposition to the underlying base rates of the two diseases. Errordriven attention theories of learning state the IBRE occurs because $\mathrm{C}$ attracts more attention than B. On the basis of this account we predicted and observed the occurrence of brain potentials associated with visual attention: a posterior Selection Negativity, and a concurrent anterior Selection Positivity, for C vs. B in a posttraining test phase. Error-driven attention theories further predict no Selection Negativity, Selection Positivity or IBRE, for control symptoms matched on frequency to $\mathrm{B}$ and $\mathrm{C}$, but for which there was no shared symptom (A) during training. These predictions were also confirmed, and this confirmation discounts alternative explanations of the IBRE based on the relative novelty of B and C. Further, we observed higher response accuracy for $\mathrm{B}$ alone than for $\mathrm{C}$ alone; this dissociation of response accuracy $(\mathrm{B}>\mathrm{C})$ from attentional allocation $(\mathrm{C}>\mathrm{B})$ discounts the possibility that the observed attentional difference was caused by the difference in response accuracy.

Keywords: EEG, visual attention, learning, categorization 


\section{Introduction}

We seem to learn more about events for which our initial predictions were incorrect than we do about events for which our initial predictions were correct - the element of surprise seems conducive to learning (Kamin, 1969). Using an eventrelated potential methodology, Wills et al. (2007) provided evidence that one process underlying this phenomenon is the rapid re-direction of visual attention in response to prediction errors. Specifically, Wills et al. (2007) found a brain potential previously associated with attention to features (e.g. shape, color, spatial frequency) - the selection negativity ( $\mathrm{SN}$ ) — for a cue involved in multiple prediction errors, relative to an equally frequent control cue involved in fewer prediction errors. In the current article, we report that a comparable event-related component is observed in the inverse base-rate effect - a robust non-rational preference observed in post-categorylearning decision making (Medin \& Edelson, 1988).

The inverse base-rate procedure, in its canonical form, can be considered both as a category-learning phenomenon (because it involves inference from learned items to unseen items, see Pothos \& Wills, 2011), and a predictive learning phenomenon (because it involves learning to predict outcomes on the basis of presented stimuli). For this reason, we use the terms 'predictive learning' and 'category learning' interchangeably in the current article, although we accept that they are not entirely synonymous when considering the associative- and category- learning literatures in their entirety (see e.g. Bott, Hoffman \& Murphy, 2007).

In the sections that follow, we describe the inverse base-rate effect, explain how the effect may be accommodated by theories of error-driven attention, and justify our prediction of the presence of a SN on the basis of these theories and related work. An experiment testing this prediction is then reported. 


\subsection{The inverse base-rate effect}

Imagine the following fictitious scenario. You are a physician in training who has just seen a series of patients. You have noticed that all patients with the symptoms dizziness and skin rash have Jominy fever, whilst all patients with dizziness and back pain have Phipp's syndrome. You have seen three times as many cases of Jominy fever as you have of Phipp's syndrome. The next patient you see has back pain and skin rash. Is this patient more likely to have Jominy fever or Phipp's syndrome?

When posed the question in this manner, people typically answer that Jominy fever is more likely (Johansen et al., 2007). Such an answer is not unreasonable because, in the microcosm of this scenario, skin rash perfectly predicts Jominy fever, and back pain perfectly predicts Phipp's syndrome, but Jominy fever is more common overall. Indeed, medical students are often encouraged to heed the aphorism "when you hear hoof beats behind you, don't expect to see a zebra" (Imperato, 1979). In the presence of two perfectly predictive but conflicting symptoms, the underlying base rates of the diseases provide one basis on which to make a decision. The current article focuses on the opposite result where participants respond that a patient with back pain and skin rash is more likely to have the rare disease Phipp's syndrome. This non-rational response bias is robustly found when participants are presented with the same information sequentially as a series of cases (e.g. Juslin et al., 2001; Kruschke, 1996; Lamberts \& Kent, 2007; Medin \& Edelson, 1988; Sherman et al., 2009).

One class of theory of this inverse base-rate effect (IBRE) is that it is a relativenovelty effect (Binder \& Estes, 1966). This theory combines the idea that novel or surprising events are particularly memorable (Rhetorica ad Herennium, 85BC; Von Restorff, 1933), with the availability heuristic (Tversky \& Kahneman, 1973), which 
states that memorable events are judged more probable. The idea that the IBRE is driven by the relative novelty of the two diseases is disconfirmed by the fact that participants predict the common disease if presented with just the symptom common to both diseases (dizziness), a response that is consistent with the underlying base rates. Participants also predict the common disease if presented with all three symptoms (dizziness, skin rash and back pain; see e.g. Kruschke, 1996); this response is also consistent with the underlying base rates and inconsistent with a relative disease novelty account of the IBRE.

Another variant of the relative-novelty explanation of the IBRE focuses on the relative novelty of the symptoms. The symptom back pain is relatively novel in this scenario compared to skin rash, which makes it more memorable, and hence its associated disease (Phipp's syndrome) is judged more probable. However, this version of a relative-novelty account is disconfirmed by the observation that the IBRE is only observed if there is a shared cue during training (Kruschke, 2001a; Medin \& Edelson, 1988; Medin \& Robbins, 1971). The shared cue in the above example is dizziness, which occurs in all presented cases. If the shared cue is replaced by further perfectly predictive cues, base-rate following is observed. For example, if dizziness and skin rash predict the common disease Jominy fever, but ear ache and back pain predict the rare disease Phipp's syndrome, then participants' modal response to the symptom combination skin rash and back pain is now Jominy fever, in agreement with the underlying base rates. Under a relative novelty account, the IBRE should still be observed, because back pain is more novel than skin rash. In summary, the sharedcue effect disconfirms the relative-novelty account of the IBRE.

The shared-cue effect also disconfirms the eliminative-inference account suggested by Juslin et al. (2001). For an extended discussion of this point see 
Kruschke (2001a) but, in essence, the eliminative-inference account proposes that participants are more likely to remember what skin rash predicts than what back pain predicts because they see skin rash more often. Faced with novel symptom combination skin rash and back pain, participants may therefore forget what back pain predicts (the rare disease) but remember what skin rash predicts (the common disease). However, skin rash plus back pain is a novel symptom combination and participants are assumed (under eliminative inference theory) to respond to this novel combination with a novel response. Specifically, they respond that skin rash and back pain predict the rare disease, because this is a novel response (responding "common disease" would be the familiar response because it is brought to mind by the more frequent symptom skin rash). Such a theory applies equally in the presence or absence of a shared cue, yet the IBRE effect depends on the presence of a shared cue. Hence, the shared-cue effect disconfirms the eliminative-inference account of the IBRE.

--- Table 1 about here, please ---

Although the above examples of the IBRE involve verbal descriptions of symptoms within a fictitious medical scenario, the IBRE has also been observed with abstract pictorial stimuli, and in non-medical scenarios (Binder \& Estes, 1966; Johansen et al., 2010; Lamberts \& Kent, 2007; Kalish, 2001; Sherman et al., 2009). We therefore subsequently discuss the IBRE and the shared-cue effect in terms of their abstract structure, which is summarized in Table 1. In Table 1, A is the shared cue, $\mathrm{B}$ and $\mathrm{D}$ are perfect predictors of the common disease com, C and $\mathrm{E}$ are perfect predictors of the rare disease rare, and $\mathrm{F}$ and $\mathrm{G}$ are further perfect predictors whose main role is to replace the shared cue. The result that the rare outcome is more likely to be predicted than the common outcome in response to a particular cue combination can be represented as: rare $>$ com. Thus, the three key results of the IBRE and 
shared-cue effect, expressed in terms of the abstract design of Table 1 are (1) com < rare for $\mathrm{BC}$, (2) com > rare for $\mathrm{DE}$, and (3) com > rare for $\mathrm{A}$. In interpreting Table 1 , it is important to note that compounds (e.g. AB) are presented simultaneously - in other words, the two component cues (e.g. A and B in AB) appear on the screen at the same time. It is also important to note that trial order is randomized, and thus the order of the rows in Table 1 is arbitrary.

\subsection{Error-driven attention}

Certain error-driven attention theories of learning (e.g. Kruschke, 2001b) can accommodate both the IBRE and the shared-cue effect. These theories are expressed in mathematical terms but, for current purposes, a natural-language approximation (Wills \& Pothos, 2012) will suffice. The central concept behind these theories of error-driven attention is that people re-direct their attention to particular components of a presented stimulus in order to minimize future prediction errors. In the context of the IBRE, one has to make the additional assumption that participants learn more quickly about what predicts the common outcome than about what predicts the rare outcome. Such an assumption is not unreasonable given that participants see the common disease more often, and it is supported by previous studies of the IBRE (e.g. Kruschke, 1996, Figure 1).

In approximate terms, the explanation provided by error-driven attention theory on the basis of these premises is as follows. Relatively early in the case series, participants learn $\mathrm{AB} \rightarrow c o m$. This leads them to initially predict $\mathrm{AC} \rightarrow c o m$, because of the similarity of $\mathrm{AC}$ to $\mathrm{AB}$. The participant's prediction turns out to be wrong, because $\mathrm{AC} \rightarrow$ rare. The participant concludes that it was cue A that led to this 
erroneous prediction (nothing has been learned about $\mathrm{C}$ yet). Error-driven attention theory states that people act to reduce the likelihood of a subsequent error in predicting the outcome of $\mathrm{AC}$ by reducing the attention paid to A and increasing the attention paid to $\mathrm{C}$. The cue $\mathrm{B}$ does not see a corresponding increase in attention, because the participant has already learned $\mathrm{AB} \rightarrow c o m$. When $\mathrm{AB}$ was originally learned, the participant knew nothing about A or B, so any initial errors would not lead to B being differentially attended relative to A.

When subsequently asked about the cue combination $\mathrm{BC}$, these error-driven changes in attention are assumed to persist, and thus $\mathrm{C}$ attracts more attention than $\mathrm{B}$. This difference in attention is presumably sufficiently large that $\mathrm{C}$ (which is associated with the rare disease) dominates the decision. Note that this explanation of the IBRE, like the IBRE itself, depends on the presence of the shared cue A. In the absence of A, base-rate following is expected because there is no shared cue to cause the re-direction of attention, and the participant has had more opportunity to learn about D than E, because D occurs more often. For similar reasons, A presented alone leads to the common outcome being predicted, because A has been followed by com more often than it has been followed by rare.

\subsection{Correlates of selective attention}

An extensive literature on the ERP correlates of selective attention to perceptual attributes has revealed a number of brain potentials elicited or modulated by attention. In the visual modality these include the $\mathrm{P} 1$ and N1 peaks of the ERP waveform, and the difference potentials N2pc, Selection Negativity (SN) and Selection Positivity (SP) obtained by subtraction of the ERPs for unattended/non-target stimuli from those for attended/target stimuli (for reviews see Hillyard \& Anllo-Vento, 1998; Luck et al., 
2000). Of particular relevance for the current study are the potentials elicited by nonspatial attention (attention to features such as color, shape and spatial frequency): $\mathrm{SN}$, which has a posterior scalp distribution and is sometimes preceded or accompanied by SP over the anterior scalp (cf. Anllo-Vento \& Hillyard, 1996). Feature-based attention can also elicit the $\mathrm{N} 2 \mathrm{pc}$ potential, but this requires lateralized presentation of target features, which was not a feature of the current study, hence the present focus on SN and SP. Importantly, SN has been previously observed in response to a cue involved in multiple prediction errors relative to an equally frequent cue involved in fewer prediction errors. Specifically, Wills et al. (2007) employed a forward cue competition design in which participants first learned that cue A predicted the presence of a disease, whilst cue B predicted the absence of that disease. Participants subsequently learned that cue combination AX predicted the presence of the disease and that cue combination BY also predicted the presence of the disease (filler cues predicted the absence of the disease in this phase). The critical part of this design is that participants tend to make fewer prediction errors on AX than on BY. By the end of this second training phase, cues $\mathrm{X}$ and $\mathrm{Y}$ have thus been presented an equal number of times, but $\mathrm{Y}$ has been involved in more prediction errors than $\mathrm{X}$. In a subsequent test phase in which $\mathrm{X}$ and $\mathrm{Y}$ were presented singly in the absence of feedback, Wills et al. (2007) found a SN for Y relative to X. This result is consistent with the idea that the difference in prediction errors led to a difference in attention to $\mathrm{Y}$ compared to $\mathrm{X}$, a difference that persisted beyond the training phase and outside the context of the specific training stimuli (AX and BY).

The current study employed the same shape-based stimuli as Wills et al. (2007) in an IBRE procedure (see Table 1). As previously stated, certain error-driven attention theories (e.g. Kruschke, 2001) predict that, after training on $\mathrm{AB} \rightarrow \mathrm{com}$ and 
$\mathrm{AC} \rightarrow$ rare, $\mathrm{C}$ will be more attended than $\mathrm{B}$. Given the evidence that the attentional changes predicted by error-driven attention theory can be indexed by a SN (Wills et al., 2007), our prediction was that a SN would be observed to cue C relative to cue B. In addition, the same error-driven attention theories (e.g. Kruschke, 2001) predict that after training on $\mathrm{FD} \rightarrow$ com and $\mathrm{GE} \rightarrow$ rare, $\mathrm{D}$ and $\mathrm{E}$ will not be differentially attended, due to the absence of a shared cue (the shared cue A being the thing that drives attention towards $\mathrm{C}$ on $\mathrm{AC}$ trials, according to these theories). We therefore also predicted that no SN should be observed for E relative to D. Cues E and D thus provide a frequency-matched control for the $\mathrm{C}-\mathrm{B} \mathrm{SN}$. Confirmation of these predictions would provide further support for these error-driven attention theories of the IBRE.

In the IBRE procedure, and as described in Table 1, during training the cues are presented solely as part of compounds. For our stimuli one could not separate the ERP effects elicited by individual cues within a compound; other methodologies, particularly eye-tracking, are better suited for achieving such separation (Kruschke et al., 2005). Hence, as in Wills et al. (2007), an EEG analysis of the training phase of the current study would be uninformative for the hypotheses under test. We therefore did not perform an EEG analysis of the training phase (for further discussion of this decision, see the General Discussion). Instead, following Wills et al. (2007), we examined the ERPs in a test phase during which the cues B and C (and D and E) were presented individually, and the SN computed as the difference waveform of these two trial types. Subsequent testing of singly presented cues avoids the difficulty of attempting to detect within-stimulus attentional allocation with an ERP methodology, and provides a particularly stringent test for error-driven attention theories. Such theories hypothesize that attentional allocation is persistent and should be observable 
outside the original training context. This issue is returned to in more detail in the General Discussion.

One shortcoming of Wills et al. (2007), and of a number of other related experiments (Beesley \& Le Pelley, 2011; Le Pelley, 2010; Le Pelley et al., 2011, in press; Livesey, Harris, \& Harris, 2009), is that the cues expected to differ in attentional allocation showed a corresponding difference in response accuracy during the test phase (or, in the case of Le Pelley et al., in press, the difference is not directly measured but can be inferred). In other words, the cue predicted to be more attended was also responded to more accurately than the cue predicted to be less attended. As discussed by Wills et al. (2007), this leaves open the possibility that we pay more attention to those things for which we already know the answer; in other words, attention is a consequence of learning. Although this is not an unreasonable hypothesis, error-driven attention theory assumes that changes in attention lead to differences in rate of learning (and hence accuracy), not (just) the other way around.

One useful feature of the inverse base-rate design is that it provides the potential for dissociating response accuracy from predicted attentional allocation. As previously discussed, error-driven attention theory predicts that $\mathrm{C}$ will be more attended than B. However, cue B is also presented more often than cue C during training so it seems possible that, in a subsequent test, participants would be more accurate on B presented alone than on $\mathrm{C}$ presented alone. Thus, in the inverse baserate design, one might expect to see a dissociation of response accuracy $(\mathrm{B}>\mathrm{C})$ from attentional allocation $(\mathrm{C}>\mathrm{B})$. If this dissociation is observed, then it seems unlikely that our results could be explained by the idea that people attend to things for which they already know the answer. Such a pattern of results would increase support for an error-driven attention account of the IBRE. 


\subsection{The current study}

We employ the basic paradigm of Wills et al. (2007) to implement an inverse base-rate training design (see Table 1), followed by a subsequent test phase that includes the critical test items B, C, D and E, presented individually. These four individually presented cues are the focus of the event-related potential analysis. The test phase also includes the behavioral test items A, BC and DE, which behaviorally test for the presence of the IBRE and the shared-cue effect in participants' responding. The behavioral test items are critical for establishing the presence of the IBRE in our study, but are uninformative in terms of the EEG analysis. In particular, and as previously discussed, it is not possible in our procedure to detect attention within stimulus compounds - for example, to distinguish between attention to B and attention to $\mathrm{C}$ when stimulus compound $\mathrm{BC}$ is presented. Thus, these behavioral test items do not form part of the event-related potential analysis (there are, in any case, insufficient trials for a meaningful ERP analysis of the behavioral test items, which were presented less frequently than the ERP test items in order to reduce overall session length). ERP test items $\mathrm{B}$ and $\mathrm{C}$ also serve as a behavioral test of the relative response accuracy of these two cues, where the result B $>$ C for accuracy, if found in conjunction with a Selection Negativity for $\mathrm{C}$ relative to $\mathrm{B}$, would be incompatible with an account of attention as a mere consequence of superior learning, thus providing support for an error-driven attention account of the IBRE.

The event-related potential methodology requires a large number of test trials, and performance should ideally be stable over the test period. In order to achieve this, we tripled the number of cues relative to the abstract design shown in Table 1. Thus, A represents three distinct cues (A1, A2, A3), similarly for $\mathrm{B}$, and so on. A review of 
the literature on the IBRE reveals that doubling or tripling the number of cues is common practice (Bohil et al., 2005; Juslin et al., 2001; Lamberts \& Kent, 2007; Kalish, 2001; Kruschke, 1996; Medin \& Bettger, 1991; Medin \& Edelson, 1988; Shanks, 1992; Sherman et al., 2009; Wood \& Blair, 2011; Winman et al., 2005). A review of the same literature also reveals that the frequency difference between common and rare outcomes is smaller in the current study than in previous reports of the IBRE (the most typical ratio is $3: 1$, the ratio in the current study was $2: 1$ ). We were thus taking a calculated risk that we would not observe the IBRE in our study. We considered this to be a risk worth taking, as it reduced the overall length of an already-long experimental session.

Another aspect of the current design that was unusual for studies of the IBRE is that further training trials were interspersed within the test phase; this technique was also employed by Wills et al. (2007), and it helps maintain stable performance across a necessarily long test phase. The current study also used abstract shapes, rather than the more typical symptom names. Abstract forms have been used successfully in a previous demonstration of the IBRE (e.g. Lamberts \& Kent, 2007). Abstract shapes were employed here, and in Wills et al. (2007), in order to elicit the brain potential associated with attention to the shape/spatial frequency of the stimulus.

\section{Materials and Methods}

\subsection{Participants and apparatus}

Eighteen right-handed undergraduate students from Exeter University (age range: 19-29 years; modal age: 20 years; 9 female, 9 male) participated on a voluntary basis. Stimulus presentation and response collection was via a PC and the 
E-prime package (Version 1.1, Psychology Software Tools, Pittsburgh, USA). The electroencephalogram (EEG) was recorded from $64 \mathrm{Ag} / \mathrm{AgCl}$ electrodes embedded in an elastic headcap (ElectroCap International, Eaton, OH, USA) connected to Brain Amp amplifiers (Brain Products, Munich, Germany). There were 58 scalp electrodes, placed in an extended 10-20 configuration; one electrode was placed on the outer canthus of each eye, one below and one above the right eye and one on each earlobe. The EEG and EOG were sampled at $500 \mathrm{~Hz}$ with a $0.016-100 \mathrm{~Hz}$ bandpass, the online reference at $\mathrm{Cz}$ and ground at $\mathrm{AFz}$.

\subsection{Stimuli}

Twenty-one abstract pictures were selected from a pool of 36 items employed in several previous studies (Jones et al., 1998; Wills et al., 2007; Wills \& McLaren, 1997; Wills et al., 2000; the pool of items is most clearly illustrated in Jones et al., 1998, Figure 1), colored red with a yellow outline, and presented against a black background. The pictures were $0.64^{\circ}$ of visual angle in diameter, presented inside a white outline square $2.5^{\circ}$ in visual angle. On trials where two pictures were presented, they were vertically aligned, one appearing $0.36^{\circ}$ of visual angle above the midpoint, and the other an equivalent distance below. On trials where one picture was presented, it was positioned in the center of the square.

\subsection{Procedure}

Participants were asked to imagine that they worked for a medical referral service, and that their job was to predict which of two fictitious diseases ("Jominy Fever" or "Phipp's Syndrome") each patient had contracted, on the basis of "cell bodies" in their blood samples (represented by abstract pictures). The allocation of the 
labels Jominy Fever and Phipp's Syndrome to the common and rare disease was counterbalanced across participants. The 21 pictures of cell bodies were, separately for each participant, randomly divided into seven cell types (three cell bodies each) corresponding to the stimulus types $\mathrm{A}-\mathrm{G}$ in Table 1 . Hence, there were three instantiations of basic structure shown in Table 1; with each letter in the table representing three randomly selected cell bodies. The same two fictitious diseases (Jominy Fever and Phipps Syndrome) were used for all three instantiations of the abstract design.

--- Figure 1 about here, please ---

The structure of each trial is illustrated in Figure 1. Trials began with the presentation of an outline square. After $1 \mathrm{sec}$, one or two "cell bodies" appeared inside the square. Participants were expected to make either a "Jominy" or a "Phipp's" response by pressing one of two keys on a standard PC keyboard. Allocation of "Jominy" and "Phipp's" responses to these two keys was counterbalanced across participants. Once the participant had responded, the abstract pictures and outline square were replaced with a feedback message that indicated whether the participant's response was correct or incorrect, and also indicated the correct response. If no response was made within $2 \mathrm{sec}$ of the onset of the "cell bodies", the screen cleared and the message "Out of Time-Please Speed Up!" was presented for $1.5 \mathrm{sec}$. The next trial followed immediately after this message. In the test phase of the experiment, test trials were followed by the uninformative feedback message "????-DATA MISSING".

The experiment had two phases, a training phase, followed by a test phase. Trial order within each phase was randomized within each of several latent sequential blocks; starts of blocks were not signaled to participants in any way. Block length was 
18 trials for the training phase, with $\mathrm{AC}$ and GE trial types each occurring three times per block, and $\mathrm{AB}$ and $\mathrm{FD}$ trial types each occurring six times per block. Block length in the test phase was 51 trials - the 18 trials of a training block, plus 33 test trials for which feedback was uninformative. The 33 test trials comprised six presentations of each of the B, C, D and E stimulus types, plus three presentations of each of the A, $\mathrm{BC}$ and $\mathrm{DE}$ trial types. There were 20 blocks in the training phase and 8 blocks in test phase; thus, the training phase comprised a total of 360 trials whilst the test phase comprised a total of 408 trials. Each of the three abstract pictures within any given stimulus group (i.e. $\mathrm{A}-\mathrm{G}$ ) occurred equally often in each block.

\subsection{Electrophysiological analysis}

Offline, the EEG was low-pass filtered at $40 \mathrm{~Hz}$ (24 dB/oct.), re-referenced to the averaged ear channels and segmented into $600 \mathrm{~ms}$ epochs, comprising $500 \mathrm{~ms}$ post-stimulus onset plus $100 \mathrm{~ms}$ pre-stimulus baseline. Following baseline correction, all epochs were inspected for ocular, muscle, movement and other artifacts and the contaminated epochs discarded. The remaining epochs were averaged, collapsing across response type (Jominy, Phipps) to yield the ERPs for the four stimulus types of interest: B, C, D and E.

We aimed to analyze the ERPs in a manner that was both comprehensive and specific, whilst controlling the rate of false positives in multiple tests (Type 1 error). To achieve this, we employed a two-stage procedure. The first stage focused on "temporal scanning" of ERPs for any differences between the trial types of interest (B vs. C; D vs. E) using a spatially non-specific technique that controls the likelihood of Type 1 error. The second stage ascertained the presence of spatially circumscribed effects such as SN with a more spatially specific ANOVA-based analysis and tested 
for the critical interaction between condition (experimental vs. control) and frequency (high vs. low).

In the first stage, in order to examine the entire ERP waveform for potential differences between trial types, the ERPs were submitted to Topographic Analysis of Variance (TANOVA; Pascual-Marqui et al., 1995), which examines the differences between conditions not at the level of individual electrodes or groups of electrodes, but at the level of entire scalp distributions (maps). As a measure of "global" dissimilarity, it is well suited for testing multiple time ranges, because it reduces the problem of correction for inflation for Type 1 error in multiple tests from two dimensions (time $\mathrm{x}$ space) to one dimension (time) ${ }^{1}$. TANOVA was run for several time-windows (hence the need to control Type 1 error, see footnote). These time windows were determined by inspecting the difference map between conditions (i.e. the scalp distribution of the $\mathrm{C}-\mathrm{B}$ difference wave), identifying the points of large changes in the scalp distribution and defining the intervals of relative topographic stability between these points as the intervals to be analyzed. Because one would not expect the current manipulations to affect very early sensory ERPs (latency $<50 \mathrm{~ms}$ ), time windows were defined in the $50-500 \mathrm{~ms}$ post-stimulus-onset range. Relative to

\footnotetext{
${ }^{1}$ TANOVA treats the scalp map of each condition as a vector defined by the scalp electrodes (58 in the present analysis). Since the difference between the vectors of two experimental conditions (e.g. C - B difference map) is also a vector, one can compute the magnitude of this difference map as the square root of the sum of squared differences between conditions at each electrode (the length of the difference vector). To assess the statistical significance of this difference, we used 5000 random permutations - this provides robust, if somewhat conservative, control for Type 1 error (Nichols \& Holmes, 2002) in performing TANOVA tests repeatedly over the entire length (duration) of the ERP. TANOVA has been used successfully in previous cognitive ERP paradigms (cf. Lavric, Forstmeier, \& Pizzagalli, 2004; Lavric, Mizon, \& Monsell, 2008).
} 
performing TANOVA across all time points (cf. Lavric et al., 2008), defining intervals in this way increases statistical sensitivity because it reduces considerably the number of tests; note that the temporal autocorrelation of ERP data also renders correction for multiple point-by-point tests (see Footnote 1) somewhat conservative.

ERPs were referenced to an average-free montage (the average reference) to ensure that the contributions of individual electrodes to the TANOVA calculations were not determined by their spatial relation to the reference channels (ear channels). The graphics (Figures 3 and 4) and ANOVA analyses (below) were based on earreferenced data. For completeness, TANOVA was also run on the control pair of conditions (D and $\mathrm{E})$.

In the second stage of the analysis, the time windows for which TANOVA revealed reliable differences were submitted to ANOVAs run on the trial types of interest (B and C) along with the control trial types (D and E). Prior to ANOVAs, ERP electrodes were averaged in 12 scalp regions covering a 4 (anterior-to-posterior) x 3 (laterality) spatial matrix, see Figure 4; region and laterality were both included as factors in the ANOVA. The purpose of this grouping was to achieve an optimal compromise between spatial specificity and adequate signal-to-noise ratio through spatial smoothing, whilst also ensuring complete scalp coverage. The Huynh-Feldt correction for violations of sphericity was applied when necessary in ANOVAs (uncorrected degrees of freedom are reported).

\section{Results}

Two participants failed to achieve above-chance accuracy in the training phase and were excluded from all subsequent behavioral and electrophysiological analyses. 


\subsection{Behavioral results}

Figure 2 illustrates performance across the training phase. Accuracy was higher in the final block of training than the first, $\mathrm{F}(1,15)=105.65, \mathrm{p}<.001$; higher for common $(\mathrm{AB}, \mathrm{FD})$ than for rare $(\mathrm{AC}, \mathrm{GE})$ stimuli, $\mathrm{F}(1,15)=12.76, \mathrm{p}=.003$, and lower in the presence of a shared cue $(\mathrm{AB}, \mathrm{AC})$ than in its absence (FD, GE), $\mathrm{F}(1,15)$ $=4.66, \mathrm{p}=.047$. These factors did not significantly interact, $\max . \mathrm{F}(1,15)=2.65, \mathrm{p}=$ .125. In the final block of training, the effects of stimulus frequency remained significant, $F(1,15)=15.02, p=.001$, as did the effects of a shared cue, $F(1,15)=$ $5.12, \mathrm{p}=.039$. These two factors did not significantly interact, $\mathrm{F}(1,15)=1.43, \mathrm{p}=$ .251. The effect of a shared cue on accuracy is not unexpected (the shared cue increases associative interference) and it does not affect the interpretability of the ERP results (because they are based on difference waveforms).

For test item BC, the proportion of common-disease responses was significantly lower than the proportion of rare-disease responses, mean common-disease proportion $=.36, \mathrm{t}(15)=2.24, \mathrm{p}=.041$, indicating the presence of an inverse base-rate effect. For test item A, the proportion of common-disease responses was significantly higher than the proportion of rare-disease responses, mean $=.69, \mathrm{t}(15)=4.81, \mathrm{p}<.001$ confirming that the IBRE we observed was not due to the relative novelty of the two diseases. The proportion of common-disease responses for DE was significantly higher than the proportion of rare-disease responses, mean $=.95, \mathrm{t}(15)=28.99, \mathrm{p}<$ .001 confirming that the IBRE observed was contingent on the presence of a common cue, and was not due to the relative novelty of cue $\mathrm{C}$ compared to cue $\mathrm{B}$. The proportion of common-disease responses to cue $\mathrm{B}$, mean $=.88$, significantly exceeded the proportion of rare-disease responses to cue $\mathrm{C}$, mean $=.67, \mathrm{t}(15)=2.88, \mathrm{p}=.011$, 
indicating greater response accuracy for cue B than cue C. The presence of this difference is important for the demonstration of a dissociation between attention and response accuracy. The mean reaction times were BC $731 \mathrm{~ms}$, A $835 \mathrm{~ms}$, DE $785 \mathrm{~ms}$, B $731 \mathrm{~ms}$, and C $763 \mathrm{~ms}$.

--- Figures 3 and 4 about here, please ---

\subsection{Event-related potentials.}

Figure 3 shows ERP waveforms for the conditions of interest $(B, C)$ and the control conditions $(\mathrm{D}, \mathrm{E})$ for a subset of 12 electrodes. An examination of the time course of ERP differences between conditions C and B (Figure 4, top panel) reveals several apparent effects. The earliest difference seemed to emerge at $\sim 120-170 \mathrm{~ms}$ and was characterized by a more positive voltage distribution for the $\mathrm{C}$ condition over the right-central scalp, followed at $\sim 200-250 \mathrm{~ms}$ by a central midline positivity for C. From $\sim 250-270 \mathrm{~ms}$ the positivity for $\mathrm{C}$ became more anterior and increasingly left lateralized, and was accompanied by occipital negativity on $\mathrm{C}$ trials (relative to $\mathrm{B}$ trials). This posterior negative and anterior positive distribution of the $\mathrm{C}-\mathrm{B}$ difference was stable until $\sim 320 \mathrm{~ms}$, when the anterior positivity shifted to the midline, whilst the posterior negativity remained relatively unchanged until $\sim 360 \mathrm{~ms}$. Subsequently, the posterior negativity faded whereas the anterior positivity persisted at midline until $440 \mathrm{~ms}$, after which the positivity for $\mathrm{C}$ became more centrally distributed and more widespread towards the end of the ERP epoch.

Some of the effects in the contrast between the control conditions $(E-D$, Figure 4, middle panel) seemed to resemble the $\mathrm{C}-\mathrm{B}$ differences: a right-central positivity at $\sim 120-170 \mathrm{~ms}$ and a mid-central positivity at $230-240 \mathrm{~ms}$. However, there were also marked differences, particularly after $200 \mathrm{~ms}$ post-stimulus. The anterior 
positivity ( 250-440 $\mathrm{ms})$ and the posterior negativity $(270-360 \mathrm{~ms})$ seen in the $\mathrm{C}-\mathrm{B}$ difference are not apparent in the E - D difference maps (there is instead some midcentral positivity at $\sim 270-330 \mathrm{~ms}$, followed later by mid-central negativity at $\sim 370$ $410 \mathrm{~ms})$. Overall, $\mathrm{E}-\mathrm{D}$ differences appear reduced relative to $\mathrm{C}-\mathrm{B}$ differences, particularly from $200 \mathrm{~ms}$ after stimulus onset.

\subsubsection{Stage 1 analysis}

Based on the scalp distribution of the difference waveform, seven time windows were defined and submitted to TANOVA of the C - B difference: 50-120 ms, 120$170 \mathrm{~ms}, 170-270 \mathrm{~ms}, 270-320 \mathrm{~ms}, 320-360 \mathrm{~ms}, 360-440 \mathrm{~ms}$ and $440-500 \mathrm{~ms}$ (see Figure 4, top panel). TANOVA and the permutation-based correction for multiple comparisons found the difference between the scalp maps of B and C trial types to be statistically significant in the $270-320 \mathrm{~ms}$ time window. This time window was associated with scalp distributions characteristic for the posterior selection negativity (SN) and frontal selection positivity (SP) (see Figure 4, top panel). The SN was right lateralized and the SP was left lateralized, possibly suggesting overlapping intracerebral generators; the magnitudes of the SN and SP were comparable. The differences between the $\mathrm{B}$ and $\mathrm{C}$ conditions were not significant in the other timewindows, the nearest to significance ( $p=0.11$, corrected for multiple comparisons) was the difference in the immediately following time window (320-360 ms), characterized by some persistence of the SN and a shift in the distribution of the SP to a more midline positivity. A similar set of time windows was defined for the $E-D$ difference (see Figure 4, middle panel). TANOVA found no statistically significant effects in any of these time-windows (largest $\mathrm{p}=0.3$, corrected). 


\subsubsection{Stage 2 analysis}

In order to better characterize the difference revealed by TANOVA between the B and C conditions, ERP amplitudes in the 270-320 ms time window were submitted to a condition (B vs. C) by anterior-posterior (4) by laterality (3) ANOVA. As expected the condition by anterior-posterior interaction was reliable, $\mathrm{F}(3,45)=5.66$, $\mathrm{p}=0.002$, confirming the presence of the posterior SN along with the anterior SP. The interaction between condition and laterality was nearly significant, $F(2,30)=$ $2.82, \mathrm{p}=0.075$, suggesting a tendency for the lateralization of these effects. No main effects or interactions were significant in the corresponding ANOVA comparing D and $\mathrm{E}$. In order to confirm that the B vs. C difference was not reducible to effects of the difference in their frequency, the ERP amplitudes in the two scalp regions where SN and SP were observed (left frontal and right occipital) were submitted to an ANOVA along with the corresponding regions for the control conditions D and E. The critical interaction between condition (experimental vs. control), frequency (high vs. low), and region (left frontal vs. right occipital) was statistically significant, F (1, $15)=4.72, p=0.046$

\section{Discussion}

We reported an ERP investigation of the inverse base-rate effect (IBRE), a paradoxical yet robust phenomenon in predictive learning. Participants were trained that stimulus compound $\mathrm{AB}$ predicted a frequently occurring outcome, whilst $\mathrm{AC}$ predicted a rare outcome. As expected on the basis of previous behavioral studies (e.g. Medin \& Edelson, 1988), participants inferred that BC predicted the rare outcome. This inference seems non-rational, but can be predicted by certain errordriven attention theories of predictive learning (e.g. Kruschke, 2001b). Such theories 
predict that, under conditions where there is a shared cue $(\mathrm{A})$ and where $\mathrm{AB}$ is more frequent than $\mathrm{AC}, \mathrm{C}$ will come to be more attended than $\mathrm{B}$. This difference in attention is assumed to dominate responding to $\mathrm{BC}$. On the basis of this prediction, combined with an extensive literature on the ERP correlates of selective attention (Hillyard \& Anllo-Vento, 1998), we predicted and observed a posterior selection negativity (SN), and a concurrent frontal selection positivity (SP), for C relative to B in the test phase of our IBRE procedure. The frontal SP seemed to also be present in the time-window preceding the $\mathrm{SN}$, though this effect was not statistically reliable.

We further predicted that no corresponding effect would be observed for a pair of control stimuli (D and E), which had the same relative frequency as B and C, but for which there was no shared cue during training (and hence for which no IBRE should be observed according to error-driven attention theory). These predictions were also confirmed, with participants inferring that DE predicted the common outcome, and with the E versus D difference in the ERPs being both non-significant, and significantly smaller than the $\mathrm{C}$ versus $\mathrm{B}$ difference.

The $\mathrm{SN}$ for $\mathrm{C}$ relative to $\mathrm{B}$ was observed under conditions where response accuracy for B exceeded response accuracy for C. Consequently, it appears that $\mathrm{C}$ was both the more attended stimulus and the one about which participants were less certain. This dissociation between attention and response accuracy appears difficult to explain if one assumes that the attentional differences observed merely reflect people attending to those stimuli for which they know the outcome. Such an account suffices for the only previous study of error-driven attention in predictive learning to use an ERP methodology (Wills et al., 2007), and it can also accommodate a range of results using eye-tracking and other methodologies (Beesley \& Le Pelley, 2011; Le Pelley, 2010; Le Pelley et al., 2011; Livesey et al., 2009). However, for the current results, 
such an account is disconfirmed, due to the presence of the aforementioned dissociation.

The occipital negativity we documented in response to $\mathrm{C}$ relative to $\mathrm{B}$ had a later onset than the 'classical' SN which, according to the influential review by Hillyard and Anllo-Vento (1998) emerges between 125 and 200 ms. However, the early SN literature was based on discriminating (typically) one or two basic feature(s), such as color, orientation, spatial frequency, direction of apparent motion, etc., defined a-priori and explicitly for the participant. In contrast, in our procedure participants had to discriminate the cues based on complex features with which they were not initially familiar. The onset of SNs reported for complex target object discriminations (e.g. letters/symbols, Potts \& Tucker, 2001; photographic images, Schupp et al., 2007) is at $200-250 \mathrm{~ms}$ following the stimulus onset, which is more in line with our data. The topography of the ERP in the range of the SN in these studies (with a prominent frontal positivity accompanying the occipital negativity) is also consistent with the topography we documented.

\subsection{Theories of the inverse base-rate effect}

In the current article, we employed the term "error-driven attention theory" as a natural language approximation for a class of mathematically expressed theories of learning. This was a simplification, as this class of theories is not homogenous, and different members vary in the extent to which they can accommodate the results we have presented. In the current section, we consider the application of some specific members of the class to our data.

One of the earlier formal expressions of error-driven attention theory is due to Mackintosh (1975; see also Sutherland \& Mackintosh, 1971). Mackintosh's (1975) 
formulation of error-driven attention theory (hereafter, Mack75) is not consistent with the current results because response accuracy for B presented alone exceeds that for C presented alone in our experiment. In Mack75, the associative strength for B must therefore exceed that for $\mathrm{C}$, and thus the dominant response to $\mathrm{BC}$ is predicted to be the same as that for B. This is opposite to our current study, where the dominant response to $\mathrm{BC}$ is the same as for $\mathrm{C}$ (an inverse base-rate effect). Hence, Mack75 cannot predict the presence of an IBRE in our experiment.

Kruschke's EXIT model (Kruschke, 2001b), however, can accommodate the presence of an IBRE under conditions where the response accuracy for B presented alone exceeds the response strength for $\mathrm{C}$ presented alone; this ability is illustrated in previously published simulations of the EXIT model, see Kruschke (2003, Table 1). One reason EXIT succeeds where Mack75 fails is that in the former attention affects both responding and future learning, whilst in the latter it only affects future learning. EXIT learns to direct attention toward $\mathrm{C}$ in $\mathrm{AC}$ during training, because doing so reduces the likelihood of the error that would otherwise be caused by the association of $\mathrm{A}$ to the common disease. At test, the presentation of compound $\mathrm{BC}$ leads to attention being directed towards $\mathrm{C}$, due to the similarity of $\mathrm{BC}$ to $\mathrm{AC}$. If this attentional allocation is sufficiently strong, $\mathrm{C}$ can dominate responding, leading to an IBRE. Of course, $\mathrm{BC}$ is also similar to training item $\mathrm{AB}$, but attentional re-allocation is not required to respond correctly to $\mathrm{AB}$ during training, so the overall effect is that when $\mathrm{BC}$ is presented at test, $\mathrm{C}$ is attended more than $\mathrm{B}$.

A further critical aspect of EXIT that allows it to accommodate our results is that learned attention is normalized before it exerts its effect on responding. This is important because on trials such as B and C, where only one stimulus component is presented, the presented component is fully in control of responding; it is only where 
multiple stimulus components are presented (e.g. BC) that learned attention affects the relative control those components have over responding. This permits the model to predict more accurate responding to $\mathrm{B}$ alone than to $\mathrm{C}$ alone, despite the greater learned attention to $\mathrm{C}$ than to $\mathrm{B}$. A consequence of this formulation is that, if EXIT is the correct account of the IBRE, then the Selection Negativity in the current study presumably reflects pre-normalized learned attention in the EXIT model. This might be because normalization takes time and the Selection Negativity is quite an early component. It might alternatively be because the Selection Negativity indexes activity in EXIT's gain nodes; the gain nodes represent non-normalized learned attention in EXIT, which is subsequently normalized further down the processing stream. However, in the absence of further data, further speculation would be inappropriate. In the current paper, we have focused on the class of error-driven attention learning theory exemplified by Mack75 and EXIT. An alternative class of theory is that attention is directed towards stimuli that are followed by surprising outcomes (e.g. Pearce \& Hall, 1980; Wagner, 1978). There is at least one published theory of the IBRE within this alternative framework (Shanks, 1992). As things stand, this class of account seems to suffer from the same problem as Mack75 and for the same reason - in other words, it cannot predict an IBRE under conditions where response accuracy to $\mathrm{B}$ alone exceeds that to $\mathrm{C}$ alone, because attention is assumed to affect only future learning, not responding. However, it seems entirely possible that such accounts could be modified along the lines of the EXIT model in order to accommodate this result (i.e. the addition of a process where attention affects responding, not just future learning).

Yet another type of error-driven attention theory states that attentional allocation occurs at the level of stimulus dimensions, rather than at the level of 
particular stimulus features within those dimensions. Sutherland and Mackintosh (1971), and the Generalized Context Model (Nosofsky, 1984), are examples of this class of account. Recent work by Johansen et al. (2010) suggests that feature-based attention is a better model of the IBRE than dimension-based attention.

Other than error-driven attention theories, we are not aware of any other class of explanation that can account for the results observed in the current study. The class of theory most similar to error-driven attentional theory states that prediction errors gate learning about outcomes; in other words, predictive relationships between cues and outcomes are learned to the extent that the outcomes they predict are not already well predicted (Schultz et al., 1997; Rescorla \& Wagner, 1972; Gluck, 1992; Harris, 2006). Such theories do not incorporate stimulus-based attention per se, but they can accommodate a range of attentional phenomena in learning via the additional assumption that stimuli strongly associated to an outcome attract attention. Such theories cannot accommodate the dissociation between attention and response accuracy observed in the current study. Indeed, standard versions of these theories cannot accommodate the IBRE (Markman, 1989). Some stimulus-sampling variants of these theories can accommodate the behavioral phenomena in the current study (e.g. Gluck, 1992), but they have no mechanism by which they can explain the dissociation of response accuracy from attention.

Bayesian inference provides another class of account of predictive learning (Anderson, 1991; Sanborn et al., 2010). Although there have been some attempts to accommodate the IBRE within a Bayesian framework (Anderson, 1991), such accounts struggle to accommodate the known IBRE behavioral phenomena (Kruschke, 2006). This is perhaps unsurprising given that Bayesian accounts assume human inference is approximately rational, and the IBRE appears to be a strikingly 
non-rational phenomenon. Kruschke (2006) proposes that the IBRE can be accommodated within a Bayesian framework by assuming that predictive learning is

locally rather than globally Bayesian. Specifically, Kruschke assumes the presence of a locally-Bayesian subsystem that determines attentional allocation, feeding into a subsequent locally-Bayesian subsystem that infers cue-outcome relationships on the basis of attentionally-modulated input. In the context of the IBRE, such an account has much in common with error-driven attentional theories.

Mitchell et al. (2009) state that theories such as Mackintosh (1975) and Kruschke (2001b) are usually assumed to describe the operation of an automatic linkformation mechanism; the intended implication being that such theories predict learning and attentional allocation to be automatic, uninstructable and unconscious. The position taken in the current article is that error-driven attentional theories of predictive learning are largely silent about such issues. In agreement with Mitchell et al. (2009), our position is that nothing in those theories, or in the data of the current study, discounts the rather likely possibility that the phenomena we have observed are mediated and moderated by conscious, deliberate processes.

\subsection{Critiques and limitations}

One potential criticism of the current study, and of Wills et al. (2007), is that theories of error-driven attention assume attentional re-allocation occurs during training as a result of prediction errors produced by compound cues, whilst the current methodology assesses attention toward single cues in a post-training test phase. In response, we'd argue that the current methodology provides a particularly illuminating test of such theories, because they predict (as discussed above) that attentional allocation persists beyond the original training context. The current results 
imply that this is indeed the case, providing additional support for such theories. Nevertheless, one possible topic for future research would be to examine the N2pc component (Eimer, 1996; Kiss et al., 2007; Luck \& Hillyard, 1994) with respect to stimuli BC and DE. Such an analysis was not possible in the current study because our stimuli were small and centrally positioned. This was a deliberate choice, designed to minimize eye-movement artifacts, and to maximize comparability with Wills et al. (2007). An investigation of the N2pc would require the two components of the compound cues (e.g. BC) to be left-right lateralized.

The current study focused on a test-phase analysis of ERPs locked to stimulus onset; this was because we had clear predictions about what would be observed, predictions made on the basis of previous work (Wills et al., 2007) and formal theory (Kruschke, 2001b). Those predictions were confirmed. A complementary approach to studying the electrophysiology of predictive learning is to consider training-phase ERPs locked to the onset of feedback (Luque et al., 2012; see also Moris et al., in press). This approach represents an important contribution to the study of the electrophysiology of predictive learning; it was not pursued in the current study for two reasons.

The first reason was a lack of any clear predictions concerning event-related potentials during training that would allow one to distinguish error-driven attentional theories of the IBRE from other classes of account. More generally, it is hard to see how event-related potentials to vertically-presented compound stimuli could be informative with regard to selective attention to an element of that compound (e.g., attention to $\mathrm{B}$ in $\mathrm{AB}$ or $\mathrm{C}$ in $\mathrm{AC})$. The second reason was that, even if such predictions could be derived, analysis of event-related potentials during training poses seemingly insurmountable technical issues in the current study. Performance during 
the training phase of a predictive learning study is, by definition, dynamic. Participants start the training phase at chance, and end with high levels of performance. Meaningful analysis thus requires subdivision of the training phase into a series of sub-sections for which error rate is relatively homogenous. However, given the relatively rapid rate of learning in such studies, these sub-divisions result in too few trials per sub-section for a meaningful ERP analysis. The solution to this problem employed by Luque et al. (2012) was to ask each participant to learn the same abstract structure 30 times within the same session. However, the length and complexity of our behavioral procedure (relative to Luque's) rendered this solution impractical in the current study.

For similar reasons, we did not conditionalize our analysis of stimulus-locked ERPs by response type (common disease predicted vs. rare disease predicted). For example, about one third of responses to test item $\mathrm{C}$ predicted the common disease, whilst two-thirds predicted the rare disease. Thus, there was an average of 16 trials per participant for the common-disease response to $\mathrm{C}$; insufficient for a clear ERP analysis. Although in principle it would have been possible to extend the test session, the experiment was already rather long and, as far as we are aware, current theories make no clear predictions about the outcome of such an analysis.

Turning to possible critiques of our behavioral data, it is noticeable that response accuracy at test for the perfect predictors (B and C) was lower than in some previous studies of the IBRE. For example, Kruschke (1996, Experiment 1) reports a perfect predictor response accuracy of 0.92 (when averaged across B and C), while in the current study the corresponding figure is 0.78 . However, the IBRE has been observed across a wide range of perfect-predictor response accuracies, ranging from 0.60 in Juslin et al. (2001, Experiment 3) to 0.95 in Johansen et al. (2007, Experiment 
3). The current study sits roughly in the middle of that range. Accuracy on the perfect predictors at test is presumably a function of a number of variables, including length of training and the type of stimuli employed.

As previously discussed, response accuracy for the common perfect predictor (B) exceeded response accuracy for the rare perfect predictor (C) in the current study. An informal review of the literature indicates that both B $>C$ (Bohil et al., 2005; Juslin et al., 2001; Medin \& Edelson, 1988; Winman et al., 2005) and C > B (Juslin et al., 2001; Kruschke, 1996; Lamberts \& Kent, 2007; Medin \& Edelson, 1988; Wood \& Blair, 2011) are observed in different studies of the IBRE. There is some indication that $\mathrm{B}>\mathrm{C}$ becomes more likely as mean performance on $\mathrm{B}$ and $\mathrm{C}$ increases, with no reports of $\mathrm{B}>\mathrm{C}$ in studies where mean accuracy is substantially below .8. Mean accuracy in the current study was 0.78 , and hence the presence of the $\mathrm{B}>\mathrm{C}$ pattern was not entirely unexpected. Nevertheless, further research is required to adequately determine what causes the presence of $\mathrm{B}>\mathrm{C}$ versus $\mathrm{C}>\mathrm{B}$, and whether error-driven attention theories provide the best account of the IBRE in conditions where $\mathrm{C}>\mathrm{B}$.

Finally, the SN observed in the current study is later than that observed in Wills et al. (2007). Although the two studies employed similar stimulus elements, the designs were quite different. Notably, in the current design (but not in Wills et al., 2007) certain cues are, from the outset, encountered in two different compounds (e.g. $\mathrm{A}$ in $\mathrm{AB}$ and in $\mathrm{AC})$. This may have necessitated more thorough perceptual analysis in the current study, delaying the onset of the SN relative to Wills et al. (2007).

\subsection{Conclusions}

We presented the first electrophysiological investigation of the inverse base-rate effect, a robust non-rational bias in predictive learning. Error-driven attention theory 
predicts both the presence of the inverse base-rate effect, and the presence of a corresponding attentional ERP component, in our study. It further predicts the absence of an inverse base-rate effect, and the absence of the corresponding ERP component, under conditions where there is no shared cue during training. These results were observed. No other class of theory appears able to accommodate these results. 


\section{Acknowledgements}

The authors thank Mike Le Pelley, Ian McLaren, Chris Mitchell and Haline Schendan for their helpful comments. 


\section{References}

Anderson JR. 1991. The adaptive nature of human categorization. Psychol Rev. 98:409-429.

Beesley T, Le Pelley ME. 2011. The influence of blocking on overt attention and associability in human learning. J Exp Psychol Anim Behav Process. 37:114-120.

Bott L, Hoffman AB, Murphy GL. 2007. Blocking in category learning. J Exp Psychol Gen. 136:685-99.

Binder A, Estes WK. 1966. Transfer of response in visual recognition situations as a function of frequency variables. Psychol Monogr. 80:Whole No. 631.

Bohil CJ, Markman AB, Maddox WT. 2005. A feature-salience analogue of the inverse base-rate effect. Korean J Think Prob Solv. 15:17-28.

Eimer M. 1996. The N2pc component as an indicator of attentional selectivity. Electroencephalogr Clin Neurophysiol. 99:225-234.

Gluck MA. 1992. Stimulus sampling and distributed representations in adaptive network theories of learning. In: Healy AF, Kosslyn SM, Shiffrin RM, editors. From learning theory to connectionist theory: Essays in honor of William K. Estes. Hillsdale (NJ): Lawrence Erlbaum Associates. p. 169-199.

Harris JA. 2006. Elemental representations of stimuli in associative learning. Psychol Rev.113:584-605.

Hillyard SA, Anllo-Vento L. 1998. Event-related brain potentials in the study of visual selective attention. Proc Natl Acad Sci USA. 95:781-787.

Imperato PJ. 1979. Medical detective. New York (NY): Richard Marek.

Johansen MK, Fouqet N, Shanks, DR. 2007. Paradoxical effects of base rates and representation in category learning. Mem Cognition. 35:1365-1379. 
Johansen MK, Fouquet N, Shanks, DR. 2010. Featural selective attention, exemplar representation, and the inverse base-rate effect. Psychon B Rev. 17:637-643.

Jones FW, Wills AJ, McLaren IPL. 1998. Perceptual categorization: connectionist modelling and decision rules. Q J Exp Psychol. 51B:33-58.

Juslin P, Wennerholm P, Winman A. 2001. High-level reasoning and base-rate use: Do we need cue-competition to explain the inverse base-rate effect? J Exp Psychol Learn Mem Cogn. 27:849-871.

Kalish M. 2001. An inverse base rate effect with continuously valued stimuli. Mem. Cognition. 29:587-597.

Kamin LJ. 1969. Attention-like processes in classical conditioning. In: Jones MR, editor. Miami symposium on the prediction of behavior: Aversive stimulation. Coral Gables (FL): University of Miami Press. p. 9-33.

Kiss M, Van Velzen J, Eimer M. 2007. The N2pc component and its links to attention shifts and spatially selective visual processing. Psychophysiology. 45:240-249.

Kruschke JK. 1996. Base rates in category learning. J Exp Psychol Lear. Mem Cogn. $22: 3-26$.

Kruschke JK. 2001a. The inverse base rate effect is not explained by eliminative inference. J Exp Psychol Learn Mem Cogn. 27:1385-1400.

Kruschke JK. 2001b. Toward a unified model of attention in associative learning. J Math Psychol. 45:812-863.

Kruschke JK. 2003. Attentional theory is a viable explanation of the inverse base rate effect: A reply to Winman, Wennerholm, and Juslin (2003). J Exp Psychol Learn Mem Cogn. 29:1396-1400.

Kruschke JK. 2006. Locally Bayesian learning with applications to retrospective revaluation and highlighting. Psychol Rev. 113:677-699. 
Kruschke JK, Kappenman ES, Hetrick WP. 2005. Eye gaze and individual differences consistent with learned attention in associative blocking and highlighting. J Exp Psychol Learn Mem Cogn. 31:830-845.

Lamberts K, Kent C. 2007. No evidence for rule-based processing in the inverse baserate effect. Mem Cognition. 35:2097-2105.

Lavric A, Forstmeier S, Pizzagalli D. 2004. When go and nogo are equally frequent: ERP components and cortical tomography. Eur J Neurosci. 20:2483-2488.

Lavric A, Mizon, GA, Monsell S. 2008. Neurophysiological signature of effective anticipatory task-set control: a task-switching investigation. Eur J Neurosci. 28:1016-1029.

Le Pelley ME. 2010. Attention and human associative learning. In: Mitchell CJ, Le Pelley ME, editors. Attention and associative learning. Oxford (UK): Oxford University Press. p. 187-216.

Le Pelley ME, Beesley T, Griffiths O. 2011. Overt attention and predictiveness in human contingency learning. J Exp Psychol Anim Behav Process. 37:220-229.

Le Pelley ME, Vadillo MA, Luque D. in press. Learned predictiveness influences rapid attentional capture: Evidence from the dot probe task. J Exp Psychol Learn Mem Cog.

Livesey EJ, Harris IM, Harris JA. 2009. Attentional changes during implicit learning: Signal validity protects a target stimulus from the attentional blink. J Exp Psychol Learn Mem Cogn. 35:408-422.

Luck SJ, Hillyard SA. 1994. Electrophysiological correlates of feature analysis during visual search. Psychophysiology. 31:291-308.

Luck SJ, Woodman JF, Vogel EK. 2000. Event-related potential studies of attention. Trends Cogn Sci. 4: 432-440. 
Luque D, López F, Marco-Pallares J, Càmara E, Rodríguez-Fornells A. 2012. Feedback-related brain potential activity complies with basic assumptions of associative learning theory. J Cogn Neurosci. 24. 794-808.

Mackintosh NJ. 1975. A theory of attention: Variations in the associability of stimuli with reinforcement. Psychol Rev. 82:276-298.

Markman AB. 1989. LMS rules and the inverse base-rate effect: Comment on Gluck and Bower (1988). J Exp Psychol Gen. 118:417-421.

Medin DL, Bettger JG. 1991. Sensitivity to changes in base-rate information. Am J Psychol. 104:311-332.

Medin DL, Edelson SM. 1988. Problem structure and the use of base-rate information from experience. J Exp Psychol Gen. 117:68-85.

Medin DL, Robbins D. 1971. Effects of frequency on transfer performance after successive discrimination training. J Exp Psychol. 87:434-436.

Mitchell CJ, De Houwer J, Lovibond P. 2009. The propositional nature of human associative learning. Behav Brain Sci. 32:183-198.

Moris J, Luque D, Rodríguez-Fornells A. in press. Learning induced modulation of the stimulus-preceding negativity. Psychophysiology.

Nichols T, Holmes A. 2002. Nonparametric permutation tests for functional neuroimaging: A primer with examples. Hum Brain Mapp. 15:1-25.

Nosofsky RM. 1984. Choice, similarity, and the context theory of classification. J Exp Psychol Learn Mem Cogn. 10:104-114.

Pascual-Marqui R, Michel CM, Lehmann D. 1995. Segmentation of brain electrical activity into microstates: Model estimation and validation. IEEE Tr Biomed Eng. $42: 658-665$.

Pearce JM, Hall G. 1980. A model of Pavlovian conditioning: variations in the 
effectiveness of conditioned but not of unconditioned stimuli. Psychol Rev. $87: 532-552$.

Pothos EM, Wills AJ. Introduction. In Pothos, EM, Wills AJ, editors. Cambridge, UK: Cambridge University Press. Formal approaches in categorization. p. 1-17. Potts GF, Tucker DM. 2001. Frontal evaluation and posterior representation in target detection. Cogn Brain Res. 11:147-156.

Rescorla RA, Wagner AR. 1972. A theory of Pavlovian conditioning: Variations in the effectiveness of reinforcement and nonreinforcement. In: Black AH, Prokasy WF, editors. Classical conditioning II: Current research and theory. New York (NY): Appleton Century Crofts. p. 64-99.

Rhetorica ad herennium. 85BC. London (UK): Heinemann. English Translation. Caplan H. 1954.

Sanborn AN, Griffiths TL, Navarro DJ. 2010. Rational approximations to rational models: alternative algorithms for category learning. Psychol Rev. 117:1144-1167.

Schultz W, Dayan P, Montague PR. 1997. A neural substrate of prediction and reward. Science. 275:1593-1599.

Schupp HT, Stockburger J, Codispoti M, Junghofer M, Weike AI, Hamm AO. 2007. Selective visual attention to emotion. J Neurosci. 27:1082-1089.

Shanks DR. 1992. Connectionist accounts of the inverse base-rate effect in categorization. Connect Sci. 4:3-18.

Sherman JW, Kruschke JK, Sherman SJ, Percy EJ, Petrocelli JV, Conrey FR. 2009. Attentional processes in stereotype formation: A common model for category accentuation and illusory correlation. J Pers Soc Psychol. 96:305-323.

Sutherland NS, Mackintosh NJ. 1971. Mechanisms of animal discrimination learning. New York: Academic Press. 
Tversky A, Kahneman D. 1973. Availability: A heuristic for judging frequency and probability. Cognit Psychol. 5:207-232.

Von Restorff H .1933. Uber die Wirkung von Bereichsbildungen im Spurenfeld. Psychol Res. 18:299-342.

Wagner AR. 1978. Expectancies and the priming of STM. In: Hulse SH, Fowler H, Honig WH, editors. Hillsdale (NJ): Lawrence Erlbaum Associates. Cognitive processes in animal behavior. p. 177-210.

Wills AJ, Lavric A, Croft G, Hodgson TL. 2007. Predictive learning, prediction errors and attention: Evidence from event-related potentials and eye tracking. J Cogn Neurosci. 19:843-854.

Wills AJ, McLaren IPL. 1997. Generalization in human category learning: A connectionist explanation of differences in gradient after discriminative and nondiscriminative training. Q J Exp Psychol. 50A:607-630.

Wills AJ, Pothos EM. 2012. On the adequacy of current empirical evaluations of formal models of categorization. Psychol Bull. 138:102-125.

Wills AJ, Reimers S, Stewart N, Suret MB, McLaren IPL. 2000. Tests of the ratio rule in categorization. Q J Exp Psychol. 53A:983-1011.

Winman A, Wennerholm P, Juslin P, Shanks DR. 2005. Evidence for rule-based processes in the inverse base-rate effect. Q J Exp Psychol. 58A:789-815.

Wood MJ, Blair MR. 2011. Informed inferences of unknown feature values in categorization. Mem Cognition. 39:666-674. 
Figure Captions

Figure 1. Trial structure.

Figure 2. Accuracy in the training phase as a function of stimulus type (see Table 1) and training block. Each plot point is the mean of two consecutive training blocks (blocks 1\& 2, $3 \& 4, \ldots 19 \& 20)$.

Figure 3. ERP waveforms for the conditions of interest $(\mathrm{B}, \mathrm{C})$ and the control conditions (D, E) shown for a subset of 12 electrodes; as in the actual EEG cap, lateral electrodes vary in their distances from the midline (e.g., F5, C3, O1). Inspection of the waveforms reveals from $\sim 250-300 \mathrm{~ms}$ a more negative-going ERP for $\mathrm{C}$ relative to $\mathrm{B}$ at posterior electrodes (particularly over the right hemiscalp) and more positive amplitudes for $\mathrm{C}$ relative to B over frontal electrodes (particularly over the left hemiscalp); neither effect is apparent in the D vs. E contrast (see Fig. 3 for the scalp distribution of the contrasts between the two pairs of conditions).

Figure 4. ERP contrasts presented in the upper and middle panels as spherical spline interpolated difference maps framed to represent windows used for TANOVA (the solid red frame with grey fill shows the window of reliable differences corrected for multiple tests; the broken red frame shows the window that approached significance), and in the lower panel as ERP plots for representative electrodes; a schematic of the scalp regions used in the ANOVA is also shown. 

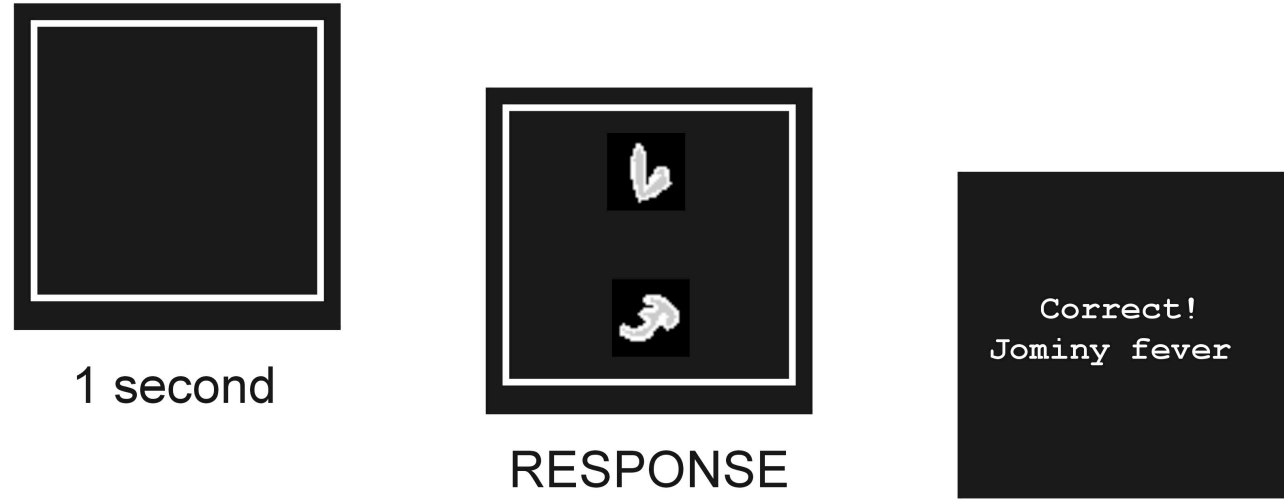

1.5 seconds 


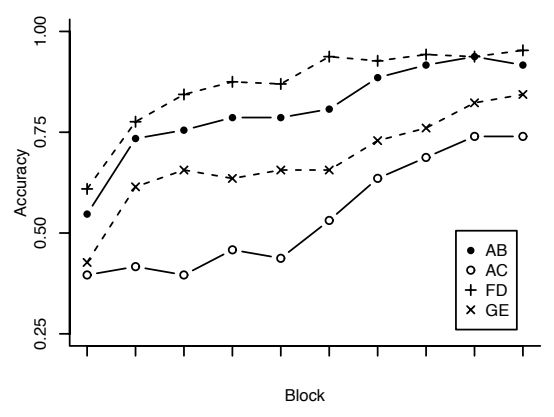



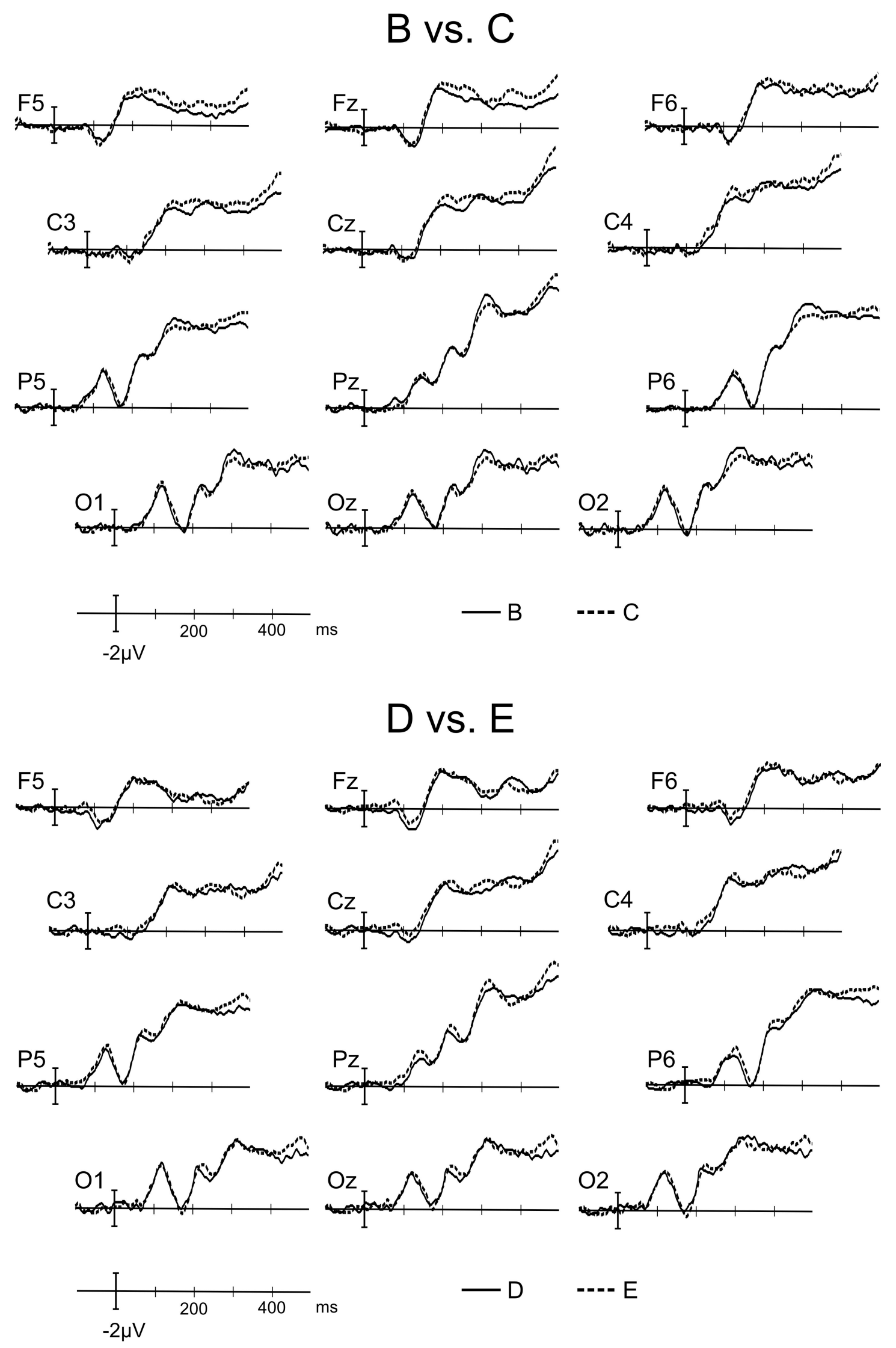

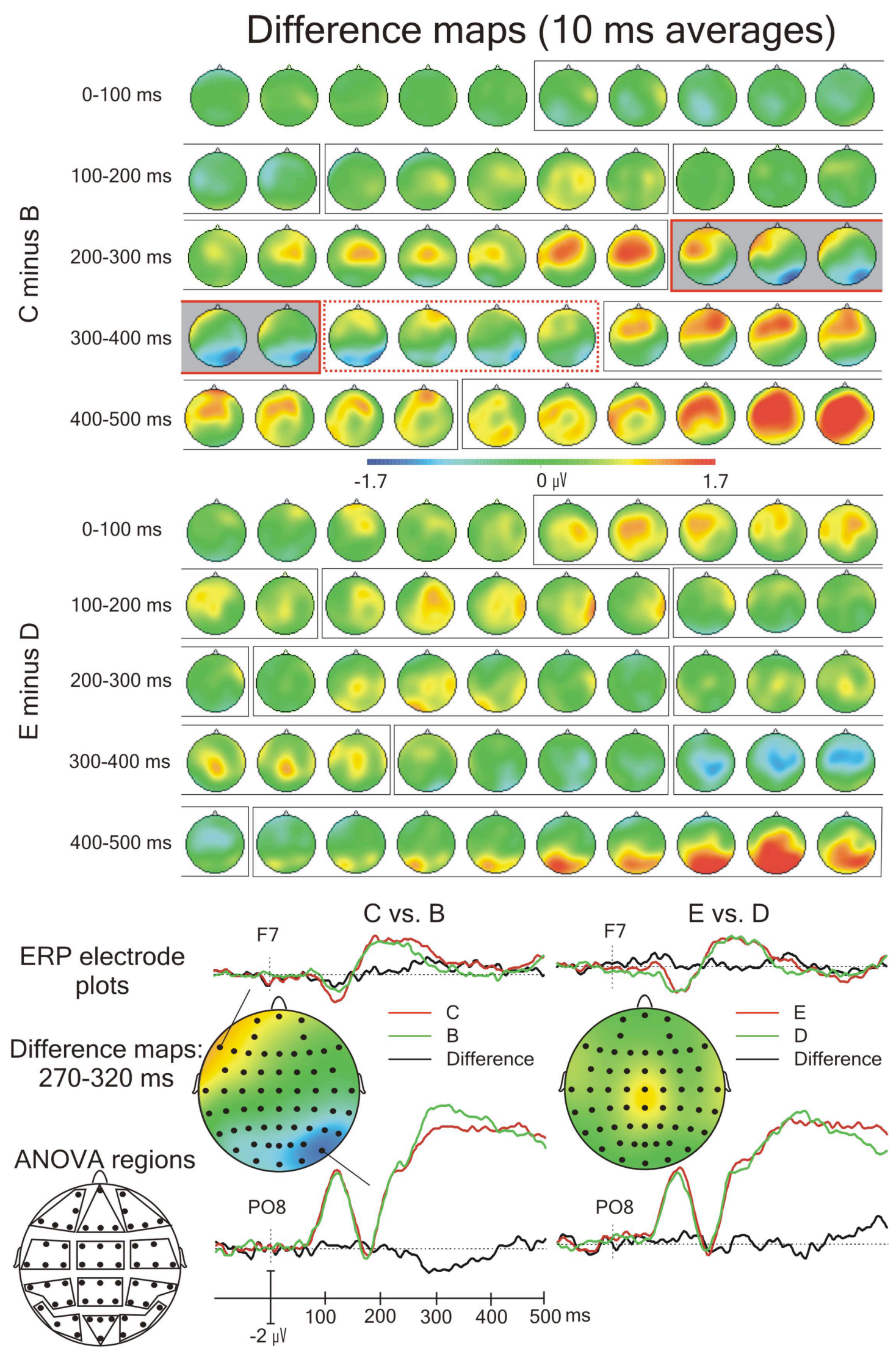